\title{
Propuesta de modelo para diagnosticar la cultura de emprendimiento sustentable en la comunidad académica. \\ Caso: Universidad de Cundinamarca, Seccional Ubaté, Colombia
}

\author{
Sandra Milena Melo-Perdomo ${ }^{1}$ \\ Universidad de Cundinamarca - Seccional Ubaté \\ smmelo@ucundinamarca.edu.co \\ Crescencio Orrego ${ }^{2}$ \\ Universidad de Cundinamarca - Seccional Ubaté \\ crescencio.orrego@gmail.com \\ Ignacio Gómez-Roldán ${ }^{3}$ \\ Universidad de Cundinamarca - Seccional Ubaté \\ ignacio.gomez19@gmail.com
}

Fecha de recepción: 19 de marzo de 2020

Fecha de aprobación: 03 de marzo de 2021

Fecha de publicación: 10 de mayo de 2021

Cómo citar este artículo / To reference this article / Comment citer cet article / Para citar este artigo:

Melo-Perdomo, S. M.; Orrego, C.; Gómez-Roldán, I. (2020). Propuesta de modelo para diagnosticar la cultura de emprendimiento sustentable en la comunidad académica. Caso: Universidad de Cundinamarca, Seccional Ubaté, Colombia. Revista Escuela de Administración de Negocios, (89), 215-232. DOI: https://doi.org/10.21158/01208160.n89.2020.2870

\section{Resumen}

Actualmente es creciente la población que reconoce la problemática de la sustentabilidad, pero el enfrentamiento del problema exige formas de pensar y actuar coherentes con los principios y valores que se deben estudiar y fortalecer en el entendido de cultura emprendedora sustentable. Por esto, el presente artículo tiene por objetivo analizar la propuesta y los resultados de aplicación de un modelo para diagnosticar en la comunidad académica la cultura de emprendimiento sustentable y la apropiación de los conceptos de emprendimiento y sustentabilidad en talleres de creatividad. Es una investigación exploratoria de carácter fenomenológico y cualitativo ordinal, con muestreo por conveniencia en la comunidad educativa de la Universidad de Cundinamarca, Seccional Ubaté. Se indagó acerca de los referentes teóricos para la construcción del modelo propuesto, la implementación de taller para el desarrollo de la capacidad creativa y

Ingeniería Financiera - Universidad Piloto de Colombia. Especialización en gerencia y administración financiera - Universidad Piloto de Colombia. Maestría en administración de empresas y dirección de proyectos - Universidad Viña del mar, Chile. Investigadora del Grupo de investigación AdCUN. ORCID: https://orcid.org/0000-0002-7699-5206

2 Lic. en Ciencias de la Educación - Universidad del Nordeste, Argentina. Magister en Educación - Universidad Cooperativa de Colombia. Especialista en Gerencia de Proyectos Educativos - Universidad Cooperativa de Colombia. Especialista en Edumática con énfasis en comunicación Electrónica - Universidad Autónoma de Colombia. Investigador del Grupo AdCun. ORCID: https:// orcid.org/0000-0003-0649-514X

3 Doctor en Análisis Económico-UNED, España. Magister en Economía - Universidad Nacional de Colombia. Especialización en Evaluación Social de Proyectos - Universidad de los Andes. Economista - Pontificia Universidad Javeriana. ORCID: https://orcid. org/0000-0002-3063-9433 
la aplicación de cuestionario en el inicio, en la salida y seis meses después a la población asistente. El propósito fue estimar el perfil de cultura emprendedora sustentable y verificar la fiabilidad del instrumento mediante el alfa de Cronbach. Se logró un diagnóstico del perfil de cultura emprendedora sustentable de la comunidad académica con mayor puntuación de la dimensión ambiental, la fiabilidad confirmada del instrumento consistencia interna- y la comprobación de la apropiación del concepto de emprendimiento sustentable de asistentes al taller.

Palabras clave: emprendimiento; sustentabilidad; emprendimiento sustentable; cultura de emprendimiento sustentable; diagnóstico de emprendimiento; desarrollo sustentable.

\title{
Proposing a model to diagnose the culture of sustainable entrepreneurship in the academic community. Case: Universidad de Cundinamarca, Seccional Ubaté, Colombia
}

\begin{abstract}
Nowadays, the group of people who recognizes the problem of sustainability is growing, but facing this problem requires ways of thinking and acting that are consistent with the principles and values that should be studied and strengthened to understand sustainable entrepreneurial culture. Therefore, this article aims at analyzing the proposal and results of the application of a model to diagnose the culture of sustainable entrepreneurship and the appropriation of the concepts of entrepreneurship and sustainability in creativity workshops in the academic community. This research is exploratory in nature, with a phenomenological and qualitative ordinal character, and uses convenience sampling from the educational community of Universidad de Cundinamarca, Seccional Ubaté. We inquired about the theoretical references for the construction of the proposed model, the implementation of a workshop for the development of the creative capacity, and the application of a questionnaire at the beginning, at the end, and six months after to the attending population. The purpose was to estimate the profile of sustainable entrepreneurial culture and to verify the reliability of the instrument using Cronbach's alpha. We managed to obtain a diagnosis of the sustainable entrepreneurial culture profile from the academic community, with the highest score in the environmental dimension, to confirm the reliability of the instrument -internal consistency-, and to verify the appropriation of the concept of sustainable entrepreneurship in workshop attendees.
\end{abstract}

Keywords: entrepreneurship; sustainability; sustainable entrepreneurship; sustainable entrepreneurship culture; entrepreneurship diagnosis; sustainable development.

\section{Proposta de um modelo para diagnosticar a cultura do empreendedorismo sustentável na comunidade acadêmica. Caso: Universidade de Cundinamarca, Sede Ubaté, Colômbia}

\section{Resumo}

Atualmente tem crescido a população que reconhece o problema da sustentabilidade, mas o enfrentamento do problema exige formas de pensar e agir coerentes com os princípios e valores que devem ser estudados e fortalecidos na compreensão de uma cultura empresarial sustentável. Por esse motivo, o presente artigo 
tem como objetivo analisar a proposta e os resultados da aplicação de um modelo para diagnosticar na comunidade acadêmica a cultura do empreendedorismo sustentável e a apropriação dos conceitos de empreendedorismo e sustentabilidade em workshops de criatividade. Trata-se de uma pesquisa exploratória de natureza fenomenológica e qualitativa ordinal, com amostragem por conveniência na comunidade educacional da Universidade de Cundinamarca, Sede Ubaté. Foram pesquisados os referenciais teóricos para a construção do modelo proposto, a realização de um workshop para o desenvolvimento da capacidade criativa e a aplicação de um questionário no início, na saída e seis meses depois à população atendente. $\mathrm{O}$ objetivo era estimar o perfil da cultura empreendedora sustentável e verificar a confiabilidade do instrumento por meio do alfa de Cronbach. Conseguiu-se um diagnóstico do perfil de cultura empreendedora sustentável da comunidade acadêmica com maior pontuação na dimensão ambiental, a comprovada confiabilidade do instrumento - consistência interna - e a comprovação da apropriação do conceito de empreendedorismo sustentável dos participantes do workshop.

Palavras-chave: empreendedorismo; sustentabilidade; empreendedorismo sustentável; cultura de empreendedorismo sustentável; diagnóstico de empreendedorismo; desenvolvimento sustentável.

\section{Proposition d'un modèle de diagnostic de culture entrepreneuriale durable pour la communauté académique : cas de l'université colombienne de Cundinamarca, section Ubaté}

\section{Résumé}

La part de la population colombienne comprenant la problématique du développement durable est en augmentation. Cependant, faire face à cette problématique nécessite des modes de pensée et d'action en cohérence avec les principes et les valeurs étudiés et renforcés par une culture entrepreneuriale durable. Cet article vise à analyser la proposition et les résultats de l'application d'un modèle de diagnostic dans la communauté académique, la culture de l'entrepreneuriat durable et l'appropriation des concepts d'entrepreneuriat et de durabilité lors d'ateliers créatifs. Cette enquête exploratoire est de type ordinale, phénoménologique et qualitative, avec échantillonnage de commodité dans la communauté éducative de l'Université de Cundinamarca, section Ubaté. Nous analyserons les références théoriques pour la construction du modèle proposé et mettrons en place un atelier de développement des capacité créatives avec l'application d'un questionnaire en début et en fin de parcours. Le but de cet article est d'estimer le profil de la culture entrepreneuriale durable et de vérifier la fiabilité de l'instrument via l'utilisation de l'alpha de Cronbach. Un diagnostic du profil de culture entrepreneuriale durable de la communauté académique a pu être établi, montrant un score élevé pour la dimension environnementale, confirmant la fiabilité de l'instrument - cohérence interne - et la vérification de l'appropriation du concept d'entrepreneuriat durable par les participants à l'atelier.

Mots-clés: entrepreneuriat; entrepreneuriat durable; culture d'entrepreneuriat durable; diagnostic de l'entrepreneuriat; développement durable. 


\section{Introducción}

Q uizás usted haya reflexionado acerca de la sustentabilidad frente a los crecimientos de la población, la producción, el consumo a nivel mundial y la dinámica emprendedora, lo cual lleva al aumento de la preocupación por garantizar la disponibilidad de recursos y la supervivencia. La toma de conciencia de la relación entre las actividades humanas y las implicaciones para el planeta reclaman que el desarrollo actual garantice la herencia de condiciones ambientales y sociales mejoradas para las generaciones venideras. Desde los negocios, es necesario redireccionar el actuar empresarial hacia la sustentabilidad, entendida con un «triple propósito»: económico-rentable, relaciones amigables con el medio ambiente y responsabilidad social.

Según la academia, es un reto «formar en emprendimiento» con estas características particulares. $\mathrm{Si}$ bien durante las décadas pasadas las universidades han promovido el espíritu empresarial en los profesionales (Castellanos, Chávez y Jiménez, 2003), el reto hoy es preparar personas competentes con el fin de direccionar y responder a las necesidades planetarias, orientando y motivando el emprendimiento sustentable.

Se han suscitado reflexiones académicas en este sentido, tal como lo expresó De Bono en su libro Ideas para profesionales que piensan, el emprendimiento debe plantearse como cultura y requerimiento de cambios de paradigmas educacionales (De Bono, 1990, como se cita en Castellanos et al., 2003). De esta manera, se otorga mayor valor agregado para que esa cultura emprendedora adopte o haga suyo el concepto de sustentabilidad como brújula principal para las empresas.
Antecedentes de estudios sobre emprendimiento sustentable se encontraron en Andalucía España-, donde se aplicó un cuestionario digital a docentes universitarios pretendiendo relacionar las actitudes de tipo ecocéntrico con el deseo de crear empresas de base sostenible. El estudio concluye lo siguiente: «Sería deseable que se produjesen estudios destinados a caracterizar a las personas o grupos que se embarcan en el desarrollo de tales aventuras empresariales» (Ruiz-Ruano y Puga, 2016, p. 20). Este aspecto respalda a la presente propuesta. Dicha investigación expresa que esta nueva vertiente que estudia el emprendimiento sustentable podría considerarse crítica para los centros de educación superior. Sin embargo, solo se aborda el concepto de emprendimiento sustentable, de manera que se relega u olvida el de cultura, que es un factor diferenciador en la presente propuesta.

En esta dirección cobra valor para la concientización y el posicionamiento práctico del concepto de emprendimiento sustentable cuando se entiende como cultura, y puede ser punta de lanza para los cambios curriculares y metodológicos que hagan pertinente la labor de la academia frente a la revolución empresarial que se espera en el futuro.

En tal sentido no se hablaría de «formar», sino de «motivar» o «desarrollar» una cultura emprendedora que requiere la búsqueda de metodologías que fomenten, promuevan y orienten a los estudiantes universitarios en el desarrollo con principios de respeto y actuación ética frente al entorno.

Esta investigación corresponde a la primera fase de un proyecto ${ }^{4}$ orientado a la formación universitaria que fortalezca la cultura en emprendimiento

4 «Formación en emprendimiento sostenible y su influencia en la migración campo ciudad». Proyecto aprobado y financiado en convocatoria interna, Universidad de Cundinamarca, 2017. 
sustentable. Tiene por objetivo analizar la propuesta y los resultados de aplicación de un modelo dirigido a diagnosticar en la comunidad académica la cultura de emprendimiento sustentable, así como a la apropiación de los conceptos de emprendimiento y sustentabilidad en talleres de creatividad.

Se demuestra con un caso específico en el que, a partir de actividades formativas innovadoras y creativas, se puede medir y fortalecer el desarrollo de la cultura en emprendimiento sustentable. El estudio se enfoca en la comunidad académica de la Universidad de Cundinamarca, Seccional Ubaté, compuesta por estudiantes, docentes, administrativos y la comunidad perteneciente a la región de influencia ${ }^{5}$ de la institución.

La estructura del presente documento se desarrolla de la siguiente manera. Primero, se indagan y presentan diferentes antecedentes académicos centrados en los desafíos que implica para la academia la formación hacia el emprendimiento, $y$, en particular, hacia la cultura de emprendimiento sustentable. En segundo lugar, se describe la metodología del modelo propuesto y del ejercicio realizado. Como tercer acápite se muestran los resultados divididos en: aplicación del instrumento para diagnóstico de cultura en emprendimiento sustentable y descripción de los perfiles encontrados, validación del instrumento por medio del alfa de Cronbach y evaluación de la apropiación de la cultura seis meses después de realizado el experimento. Finalmente, se señalan las conclusiones y posibilidades de seguimiento.

\subsection{Emprendimiento: desafío para la academia}

El reto de fomentar la cultura emprendedora sustentable como alternativa para conseguir el desarrollo local y un sano proyecto de vida puede considerarse una utopía, si se tiene en cuenta la tendencia actual de entender el emprendimiento como creación de empresas u opción de autoempleo y no como opción visionaria de futuro planetario.

Es así cómo, según lo señala Restrepo-Sánchez «Entre 2013 y 2017, en Colombia se crearon 55
848 empresas empleadoras y 210965 empresas no empleadoras o aquellas relacionadas con actividades cuenta propia como alternativa para personas que presentan problemas de empleabilidad $\gg(2018$, p. 18).

Pareciera entonces que domina la idea reduccionista del emprendimiento a la creación de empresas que, si bien es importante, también es insuficiente desde una perspectiva de sustentabilidad, así como desde otros alcances de las actitudes emprendedoras socialmente responsables.

La academia fomenta la intención emprendedora $\mathrm{y}$, aunque no es el único factor para fortalecer la empresarialidad, sí es pieza clave en el propósito de conseguirla. La premisa del emprendedor como protagonista y principal factor para el desarrollo económico del éxito de cualquier proyecto aparece de manera recurrente en la bibliografía (Acs y Amoros, 2008; Audretsch, Keilbach y Lehman, 2006; Martínez-García, Padilla-Carmona y Suárez-Ortega, 2019; Rodríguez-Ramírez, 2009).

Desde hace décadas se tiene evidencia del impacto de la educación y de cómo el talento humano competente contribuye significativamente al crecimiento económico (Barro, 1991) y es más propenso al emprendimiento (Timmons, Zacharakiz y Spinelli, 2004). De acuerdo con el perfil esperado de las escuelas y las facultades de negocios, la intervención determinante para direccionar los esfuerzos hacia el emprendimiento sustentable debería estar enfocada en cambiar la mentalidad de los potenciales empresarios.

Estudiar emprendimiento desde el enfoque de cultura es un camino adecuado para los centros educativos, aunque la reacción de algunas universidades, en buena medida, se ha limitado a crear asignaturas con ese nombre y en otras ocasiones a incorporar la palabra emprendimiento en los microcurrículos (GómezRoldán et al. 2019); de igual forma, varias escuelas de negocios siguen incorporándola en sus titulaciones (Callejón, 2009).

La Seccional Ubaté impacta directamente las instituciones educativas, la institucionalidad, los empresarios y habitantes en general de la región geográfica denominada Provincia de Ubaté y sus diez municipios. 
El cambio de mentalidad va más allá de procesos técnicos y de formación atomizada. Requiere el desarrollo de contextos favorables para generar la cultura que siembre en los futuros profesionales los rasgos propios del emprendedor impregnados de los principios de la sustentabilidad. A fin de lograrlo es fundamental que las universidades cuenten con personal docente sin prejuicios hacia las nuevas metodologías y el docente como guía. Sin embargo, el emprendimiento y la innovación son percibidos por algunos académicos tradicionales como nuevas tendencias que disminuyen la calidad educativa, ya que los vinculan, en mayor medida, con las habilidades sociales y comunicacionales (Zisis, Moya y Molina, 2017) y no como cultura.

\subsection{Hacia el desarrollo de la cultura emprendedora sustentable}

La sustentabilidad incluye un concepto tríadico que comprende las dimensiones de viabilidad económico-rentable de un negocio, el interés por el medio ambiente y la responsabilidad social (BuggLevine y Emerson, 2011; Clegg, 2014; ContrerasPacheco, Pedraza-Avella y Martínez-Pérez, 2017; Cortés-Mura y Peña-Reyes, 2015; Muñoz, 2013; Rodríguez-Moreno, 2016; Velásquez-Álvarez y Vargas-Hernández, 2012). En este contexto, la academia debe contribuir significativamente al desarrollo de competencias para superar los retos que los futuros empresarios enfrentarán en los años venideros.

La sustentabilidad es un concepto complejo que usualmente lo utilizan de forma diferente distintos autores. No obstante, los documentos en inglés suelen usar la palabra sustainability haciendo referencia en unos casos a sostenibilidad, y en otros a sustentabilidad. En castellano, algunos autores suelen utilizar el término sostenibilidad con énfasis en el objetivo económico de sostener rentabilidad a lo largo del tiempo, mientras sustentabilidad entiende las tres dimensiones. Es preciso aclarar, por ejemplo, que la Organización de las Naciones Unidas, al referirse a los objetivos de desarrollo del milenio da igual importancia a las tres dimensiones. Sin embargo, lo que interesa universalmente es la creación de valor desde una perspectiva tripartita y el impacto permanente que se genere al trabajar en las tres dimensiones.

Es así que «las principales razones para desarrollar modelos sustentables de consumo y producción son: a) la disponibilidad limitada de recursos naturales; b) el crecimiento exponencial de la población; b) el derecho por satisfacer necesidades de las generaciones actuales y futuras» (Oriol, van Klink y Rozo-Grisales, 2011, p. 10). El futuro está en manos de individuos apasionados y motivados cuyos comportamientos están cimentados en sólidas culturas emprendedoras fundamentadas en valores ambientales, sociales y económicos que crearán las tecnologías y los modelos de negocios innovadores del mañana.

Desde las miradas de la antropología social y la perspectiva de autores clásicos en el tema de cultura como Levi-Straus, Schein y Peter Senge, el concepto de cultura implica tener en cuenta cuatro factores: los procesos de aprendizaje que generan prácticas, las percepciones y los comportamientos, el pensamiento simbólico que regula la conducta y la actividad cognitiva, el lenguaje que favorece la cooperación y el intercambio de ideas, así como las herramientas del conocimiento y el saber hacer (Gómez-Roldán, 2013).

Así, el reto de la cultura en emprendimiento sustentable implica que la comunidad se apropie de principios, valores y preconcepciones que redunden en comportamientos formales e informales de las personas hacia lo económico-rentable, social y ambiental, como una tríada concebida en un sistema indivisible que inquieta a las generaciones, especialmente desde el surgimiento del Club de Roma ${ }^{6}$.

6 Movimiento de científicos, políticos y autores de reconocida importancia, quienes evalúan la sostenibilidad y sustentabilidad de los recursos que utiliza el hombre en la construcción de los modelos de crecimiento económico. 
Con la intención de generar inquietudes formales entre las naciones, la Comisión Mundial sobre el Medio Ambiente y el Desarrollo de 1983 abordó el tema cuando expresa en su informe Brundtland (ONU, 1987):

8. [...] es imposible separar las cuestiones de desarrollo económico con las de medio ambiente. Muchas formas de desarrollo extenúan los recursos del medio ambiente en los que deben basarse, y el deterioro del medio ambiente puede socavar el desarrollo económico. La pobreza es causa y efecto principal de los problemas mundiales del medio ambiente. Es, por tanto, inútil tratar de encarar los problemas ambientales sin una perspectiva más amplia que abarque los factores que sustentan la pobreza mundial y la desigualdad internacional. (pp. 17-18)

Así, entonces, el emprendimiento sustentable es pertinente en el análisis dinámico del presente y el futuro porque «el emprendimiento $[\ldots]$ para que sea de calidad debe constituirse en procesos virtuosos que generen más beneficios que costos y que potencialmente agreguen valor y sustentabilidad a la actividad» (Petit-Torres, 2007, p. 500).

Desde visiones más radicales, «el emprendimiento sustentable se centra en la preservación de la naturaleza, el soporte vital, y la comunidad en la búsqueda de oportunidades percibidas para crear productos, procesos y servicios que generen ganancias, que se consideran económicas y no económicas» (Shepherd y Patzelt, 2011, p. 142). Entre las ganancias no económicas se encuentran aquellas orientadas al desarrollo de la cultura emprendedora que crea beneficios ambientales y sociales, lo cual permite reiterar la noción de complejidad implícita en el estudio de este fenómeno.

El emprendimiento sustentable no es una opción de benevolentes. Las empresas perciben hoy la sustentabilidad como «la licencia para crecer» en el futuro (Clegg, 2014) porque, incluso, las decisiones en pro de la protección ambiental ofrecen alternativas económicamente más beneficiosas. Por ejemplo, la gestión energética puede verse reflejada en significativos ahorros y anticipa el riesgo de reglamentaciones futuras más estrictas (Clegg, 2014).
De allí que la sustentabilidad no es una alternativa, sino el camino.

La sustentabilidad responde a las expectativas de clientes preocupados por la supervivencia. Cada vez hay más consumidores que analizan el producto, su procedencia y las prácticas productivas. De allí que el marketing triple $\mathrm{P}$ - people, planet, profit - abarca los aspectos sociales, ecológicos y económicos de una compañía o producto (Pomering, Kyriazis y Johnson, 2014). Pero no todos los consumidores son iguales, algunos son más sensibles al precio mientras otros están más atraídos por el valor intangible están dispuestos a pagar precios altos-; de esta manera, al desarrollarse soluciones sustentables, los consumidores exigen crecientemente estas características en los productos y servicios.

Típicamente, los emprendedores «sustentables» tienen la misión personal de hacer del mundo un mejor lugar para las personas que los rodean $y$ aplican su imaginación a problemas retadores a fin de concebir, hacer prototipos y crear soluciones con valor ambiental, social y económico-rentable (Oriol et al., 2011).

En este orden de ideas, el ecosistema tradicional de los negocios debe integrarse en una cultura de emprendimiento sustentable, de modo que convierta la rentabilidad privada, el desarrollo social y la protección ambiental en objetivos estratégicos para beneficiar a las empresas, las localidades, los territorios, las regiones y los países, en una perspectiva integral del desarrollo.

De allí que el papel de la universidad es encaminar las generaciones futuras en este sentido y lograr premisas como la siguiente: «Entre las personas jóvenes profesionales, existe una conciencia hacia el cuidado del medio ambiente, y un interés por hallar y ejecutar soluciones a las problemáticas subyacentes; algunas de ellas se convierten en oportunidad para crear empresa» (Arroyave-Puerta y Marulanda-Valencia, 2019). Así, la universidad participa en una tendencia cultural orientada y fortalecida por las actuales escuelas de formación de profesionales. 


\section{Método}

$\mathrm{E}$ sta investigación es exploratoria de carácter fenomenológico y cualitativo. Es cualitativa ordinal porque el uso del instrumento implica que quienes participaron proporcionaron respuestas de carácter perceptual al calificarlas con variables discretas $-1,2,3,4$ y $5-$ como corresponde a la estructura semántica de likert (Hernández-Sampieri, Fernández-Collado y Baptista-Lucio, 2014). El alfa de Cronbach es un método que permite comprobar la fiabilidad de este tipo de instrumentos cualitativos. Sin embargo, el coeficiente alfa surge de promedios de coeficientes de correlación y cuando se comprueba la fiabilidad de las variables agrupadas se pueden calcular promedios que dan unas aproximaciones paramétricas para analizar los fenómenos.

Con muestreo por conveniencia se obtuvo información de treinta personas de la comunidad académica —estudiantes de primero a sexto semestre de administración de empresas, docentes y empresarios-, en un estudio de caso con pilotaje de un instrumento para medir la inclinación espontánea al incluir las variables de sustentabilidad en sus decisiones, cuando se piensa en emprendimiento. Se aplicó en un espacio académico denominado «Taller para el desarrollo de la capacidad creativa».

La empresa colombiana C-INNOVA, colaboradora del ejercicio, apropia, mejora y divulga, a través de diversas actividades — como, por ejemplo, el Taller para el desarrollo de la capacidad creativa- los prototipos tecnológicos de bajo costo que se cocrean en las múltiples cumbres internacionales de diseño y desarrollo-IDDS desarrolladas por el D-Lab del Instituto Tecnológico de Massachussets.
El espacio de reflexión-taller tiene en cuenta los siguientes aspectos de sustentabilidad: 1) materiales utilizados de fácil acceso y 2) de bajo costo; 3) el reconocimiento de recursos del medio; 4) la obligación ética de evaluar la procedencia de los insumos y las materias primas; y 5) el logro de equilibrios en las cadenas productivas. Estos aspectos fueron determinantes para seleccionar el taller que sirvió de escenario para la sensibilización que se buscaba.

El instrumento o cuestionario se aplicó a un grupo heterogéneo de personas de la comunidad académica de Ubaté. Este se construyó con la estructura semántica de Likert y veinte variables que responden a la siguiente pregunta: $\ll_{i}$ Con qué frecuencia usted lee, habla, conversa o analiza $[. .$.$] en su diario vivir$ $[\ldots]$ ?», seguida de las veinte variables-preguntas que definen las tres dimensiones de emprendimiento sustentable. Ocho de ellas corresponden a la dimensión económica, ocho a la ambiental y cuatro a la social.

El tratamiento de los datos implicó la conversión de equivalencias de la escala de Likert ordinales a escalas numéricas también ordinales con el fin de analizar los cambios, teniendo en cuenta las frecuencias, sus proporciones $y$, especialmente, para el análisis de fiabilidad con el alfa de Cronbach. La conversión de los datos, en los cinco niveles en los cuales se obtienen respuestas, se realizó bajo el criterio descrito en la tabla 1.

Tabla 1. Asignación numérica a los niveles de la escala de Likert aplicados en el instrumento

\begin{tabular}{|c|c|}
\hline Escala de Likert & Nivel -escala numérica- \\
\hline Nunca & 1 \\
\hline Raramente & 2 \\
\hline Ocasionalmente & 3 \\
\hline Frecuentemente & 4 \\
\hline Muy frecuentemente & 5 \\
\hline
\end{tabular}

Fuente. Elaboración propia. 
El instrumento fue construido fundamentándose especialmente en los estudios de los siguientes investigadores.

- Blancas-Peral et al. (2010), quienes describen las variables que son pertinentes para cada una de las tres dimensiones de sustentabilidad.

- List et al. (2017), de cuyo estudio se toman variables de la dimensión ambiental relacionadas con la protección de ecosistemas en peligro.

- Vásquez-Stanescu et al. 2017, del que se toman como referencia las variables de los temas energéticos y ambientales relacionados con la gestión empresarial.

El cuestionario se aplicó dos veces —al iniciar y al finalizar el taller- con el objeto de contrastar el cambio en la percepción de apropiación de las dimensiones antes y después de la experiencia. Al ser variables ordinales, se considera adecuada la contrastación con el uso de frecuencias interpretándose el cambio como fruto de las reflexiones colectivas generadas durante el desarrollo del taller. Si aumenta la frecuencia en los grados altos de la escala de Likert, hay mayor toma de conciencia — apropiación - sobre los fenómenos que se evalúan. Si ocurre lo contrario, se entiende, no obstante, que quien responde es más prudente al expresar su opinión y dar más importancia al fenómeno.

Este taller orientado al desarrollo de la creatividad permite medir las potencialidades en emprendimiento sustentable, ya que la creatividad, según Ken Robinson, es el proceso de tener ideas con valor que a menudo resultan de la interacción de diferentes perspectivas: «Para que una idea sea creativa, debe ser apropiada, útil y aplicable» (Amabile, 2005, p. 1).

Estas características propias de los principios y los prototipos se visibilizan y comprueban en este tipo de talleres. Además, conviene anotar que «el talento humano creativo ejerce impactos directos en el desarrollo regional» (Florida, Mellander y Stolarick, 2008, p. 616), asunto que hace pertinente aprovechar integralmente las actividades de fortalecimiento $y$ concientización de emprendimiento sustentable.

El taller integró las dimensiones social, ambiental y económica mientras se construían los prototipos. Se desarrollaron conversatorios y retos en las actividades de construcción creativa desarrolladas por los participantes durante el taller, en medio de reflexiones orientadas hacia el desarrollo de la iniciativa y la creación de empresa.

Seis meses después se aplicó una prueba de tres preguntas que indagaron sobre la apropiación del concepto de sustentabilidad en el momento de crear empresa. Esto con el objetivo de reconfirmar si luego de la sensibilización llevada a cabo en el taller los asistentes se habían apropiado del concepto -incorporando el concepto de emprendimiento sustentable como cultura-principios y valores junto con formas de pensar y actuar - en el mediano o largo plazo, o si las respuestas iniciales correspondieron a reacciones emotivas de corto plazo. Esta acción se desarrolló coherentemente con variables usadas en el instrumento inicial.

\section{Discusión y resultados}

T a primera de tres etapas de análisis agrupa las _aproximaciones enfocadas en identificar las potencialidades de la población hacia la cultura en emprendimiento sustentable. Contempla el análisis de promedios y las desviaciones estándar de cada una de las dimensiones en el estudio del fenómeno, y luego el análisis detallado de las variables. En la segunda se prueba la fiabilidad estadística del cuestionario a través del alfa de Cronbach y en la tercera se analizan las respuestas del instrumento aplicado seis meses 
después, con el objeto de ratificar si el concepto de emprendimiento sustentable se convirtió en una premisa cultural permanente.

\subsection{Perfiles de cultura en emprendimiento sustentable}

De las tres dimensiones que definen el emprendimiento sustentable, en la población observada se descubre mayor inclinación a tratar con más frecuencia las variables ambientales tanto en las repuestas de entrada - 3,43- como en las de salida - 3,42 - del taller. Esto indica que es prácticamente inexistente el cambio. Le siguen los ítems de la dimensión social, con 3,42 para la entrada y 3,29 para la salida; se nota que el indicador disminuyó y, si bien inicialmente estaba en un nivel similar al de las variables ambientales, en la salida quedó con la puntuación menor.

La dimensión económica inicia con la menor puntuación -3,34- y en la salida aumenta levemente a 3,37, pasando a segundo lugar en la puntuación. Cuando el promedio se acerca a 5, corresponde con una opción de respuesta que tiende a ser «muy frecuentemente». Si el valor es más cercano a 1 , se entenderá que la opción de respuesta tiende a «nunca» tener en cuenta las variables al momento de crear empresa (véase la Figura 1).

Figura 1. Perfil de cultura emprendedora sustentable

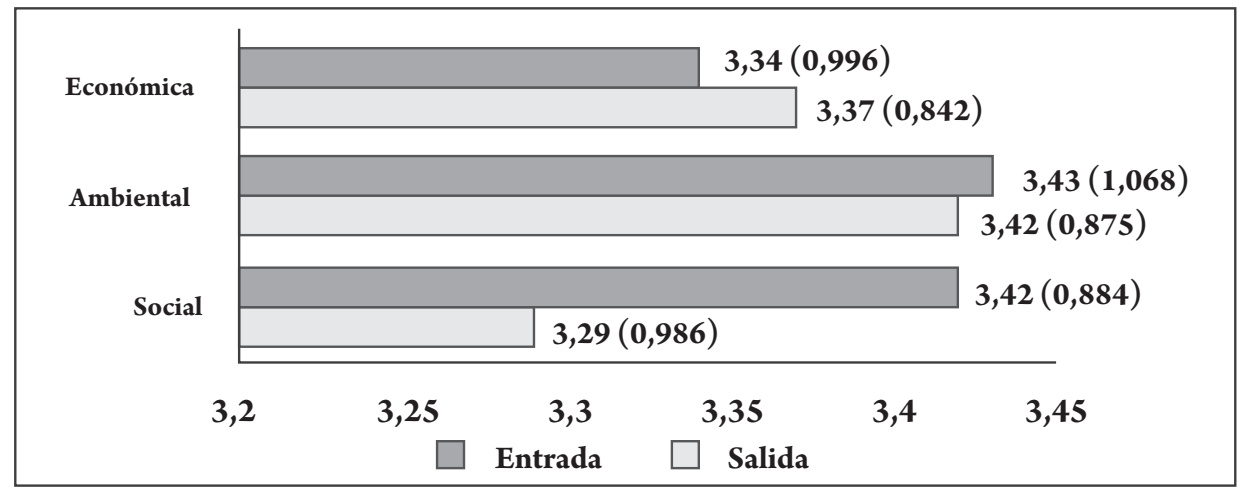

Nota. Promedio de las dimensiones a la hora de crear empresa. Respuestas a cuestionario de entrada y salida. El valor entre paréntesis corresponde a la desviación estándar.

Fuente. Elaboración propia con base en respuestas al cuestionario y el cálculo de los autores.

Los cambios de los promedios entre salida y entrada evidencian la reflexión de los asistentes sobre emprendimiento sustentable. La sensibilización lograda en el taller permite aproximarse a los cambios en las formas de pensar, decidir y actuar al tener en cuenta las tres dimensiones. Los resultados muestran que los asistentes dan mayor importancia, en su orden, a las variables ambientales, económicas y sociales. Las puntuaciones deben ser interpretadas cuidadosamente puesto que el taller implica el desarrollo de mayor sentido crítico-reflexivo y, por tanto, los asistentes al responder las preguntas en la salida del taller lo hacen con mayor prudencia.
Recordando que el valor de 1 en la escala de Likert significa «nunca» y cinco «muy frecuentemente», el aumento en la desviación estándar puede interpretarse como un alejamiento en la unidad de criterio por parte de quienes responden y las disminuciones implicarían mayor unicidad. En esta dirección, en las dimensiones ambiental y económica hay mayor coincidencia en las apreciaciones de los asistentes acerca del emprendimiento sustentable al final del taller, y lo contrario en la dimensión social (véase la Figura 1). 
Los impactos del taller en la apropiación de los conceptos de cultura emprendedora sustentable se observan al comparar los cambios en las respuestas en la entrada y la salida. Estos cambios se analizan evaluando la diferencia de modas $^{7}$, la cual se muestra en las tablas 2, 3 y 4 . En las tablas se observan cambios de una unidad $-1-$. Si la variación es positiva, hay mayor apropiación de los conceptos del fenómeno estudiado. Si es negativa, se entiende que las respuestas implican una mayor reflexión de los asistentes al expresar su posición frente a la pregunta.
Para mayor comprensión de los resultados, léase cada variable incluida en la pregunta formulada: $\ll i$ Con qué frecuencia usted lee, habla, conversa o analiza $[\ldots]$ en su diario vivir?». En los puntos suspensivos $[\ldots]$ se integra el nombre de la variable, por ejemplo: $\ll i$ Con qué frecuencia usted lee, habla, conversa o analiza la satisfacción de los residentes de una comunidad, hacia propuestas de emprendimiento, en su diario vivir?».

Tabla 2. Moda de las respuestas para cada variable en la dimensión social

\begin{tabular}{|c|c|c|c|}
\hline Variables & Entrada & Salida & Diferencia \\
\hline $\begin{array}{l}\text { Satisfacción de los residentes de una comunidad, hacia propuestas de } \\
\text { emprendimiento. }\end{array}$ & 3 & 3 & 0 \\
\hline Efectos socioculturales en la comunidad de mis propuestas de emprendimiento. & 4 & 3 & -1 \\
\hline $\begin{array}{l}\text { Acceso de los habitantes — residentes - a los principales recursos del lugar y la } \\
\text { región. }\end{array}$ & 4 & 3 & -1 \\
\hline Toma de conciencia y participación de la comunidad. & 3 & 4 & 1 \\
\hline
\end{tabular}

Fuente. Elaboración propia con base en las encuestas realizadas a los asistentes al taller y los cálculos de los autores.

Las respuestas «ocasionalmente» $\mathrm{y}$ «frecuentemente» son las más repetidas en las cuatro variables de la dimensión social. La mitad de las variables reflejan una disminución de la frecuencia, lo cual posiblemente indica el desconocimiento y la falta de internalización de aspectos relacionados con esa variable. La toma de conciencia y participación de la comunidad presenta mayor frecuencia y avances en la apropiación del concepto (véase la Tabla 2).

Tabla 3. Moda de las respuestas para cada variable en la dimensión ambiental

\begin{tabular}{|c|c|c|c|}
\hline Respuestas por niveles & Entrada & Salida & Diferencia \\
\hline Protección de ecosistemas en peligro & 4 & 4 & 0 \\
\hline Calidad de las aguas & 3 & 4 & 1 \\
\hline Gestión energética & 4 & 4 & 0 \\
\hline Disponibilidad y gestión del agua & 4 & 3 & -1 \\
\hline Tratamiento de las aguas residuales & 3 & 4 & 1 \\
\hline Gestión de los residuos sólidos & 4 & 4 & 0 \\
\hline Contaminación atmosférica & 4 & 4 & 0 \\
\hline $\begin{array}{c}\text { Gestión del impacto visual de la } \\
\text { infraestructura y las instalaciones }\end{array}$ & 4 & 4 & 0 \\
\hline
\end{tabular}

Fuente. Elaboración propia con base en las encuestas realizadas a los asistentes al taller y los cálculos de los autores.

\footnotetext{
7 Moda: estadístico que señala el número que más se repite. Para el caso, las frecuencias en la escala de Likert
} 
En la dimensión ambiental, solo en tres de las ocho variables se presentan cambios en las modas. Se ratifica lo observado en los promedios, de manera que esta es la dimensión con mayor arraigo cultural. Como hallazgo particular se tiene que los cambios se presentan solo en las variables relacionadas con el agua. Hay una importante reflexión sobre disponibilidad y uso del agua, de manera que es el único cambio negativo. Los cambios positivos reflejan la mayor internalización en los aspectos relacionados con la calidad de las aguas y el manejo de aguas residuales. También en esta dimensión las respuestas se concentran en las opciones «ocasionalmente»y «frecuentemente» (véase la Tabla 3 ).

Tabla 4. Moda de las respuestas para cada variable en la dimensión económica

\begin{tabular}{|l|c|c|c|}
\hline \multicolumn{1}{|c|}{ Respuesta } & Entrada & Salida & Diferencia \\
\hline Control de la intensidad de uso de los recursos naturales & 4 & 4 & 0 \\
\hline Integración del emprendimiento en la planificación regional y local & 4 & 3 & -1 \\
\hline Creación de empleo justo & 3 & 4 & 1 \\
\hline Beneficios económicos para la comunidad de los emprendimientos & 3 & 4 & 1 \\
\hline Creación de nuevos productos acordes a la calidad de vida & 3 & 3 & 0 \\
\hline Actividades de marketing con miras al emprendimiento sustentable & 3 & 4 & 1 \\
\hline Mantenimiento de la satisfacción del cliente & 3 & 4 & 1 \\
\hline Tener en cuenta las oportunidades que se presenten para generar emprendimiento & 3 & 4 & 1 \\
\hline
\end{tabular}

Fuente. Elaboración propia con base en las encuestas realizadas a los asistentes al taller y los cálculos de los autores.

En esta investigación la dimensión económica se enfoca en la sustentabilidad, por tanto, los cambios que se presentan son significativos, al observarse desaprendizaje en torno al concepto generalizado de rentabilidad económica, de modo que vira hacia la aprehensión de lo económico desde la sustentabilidad.

De las ocho variables, seis presentan cambios y tan solo uno de ellos es negativo: la integración del emprendimiento en la planificación regional y local. De esto se deduce un cambio positivo importante en la conciencia de los asistentes, al considerar ahora los aspectos de política pública para los emprendimientos. Los cambios positivos en las modas evidencian la evolución del concepto económico-rentable hacia la inclusión de aspectos tales como empleo justo, los beneficios económicos para la sociedad, el marketing, la satisfacción de los clientes y las oportunidades orientadas todas hacia el emprendimiento sustentable (véase la Tabla 4).

Recapitulando, de las veinte variables consideradas, la moda de 4 disminuyó y aumentó en 8 . El taller, por tanto, evidencia la apropiación y reflexión de los conceptos de emprendimiento sustentable. Los menores cambios en la dimensión ambiental, al contrastarse con los cambios en las otras dimensiones, permiten identificar las variaciones en las dimensiones social y económica. Por esta razón, al incorporar estas actividades como talleres de creatividad orientadas al emprendimiento sustentable en los planes de estudio y currículos se podría generar un impacto estructurado deseable de largo plazo desde la formación universitaria.

\subsection{Validación del cuestionario}

El coeficiente alpha de Cronbach se ha convertido en un indicador clásico en las ciencias sociales para el examen de confiabilidad de los instrumentos utilizados en investigaciones cualitativas ordinales. Las mediciones pueden tener diferentes errores que descansan en los sujetos, en los evaluadores y en las variables o ítems utilizados. Así mismo, pueden darse errores en las aplicaciones de las encuestas, en las interacciones entre las fuentes y, por supuesto, el error aleatorio, de tal manera que la evaluación de la consistencia interna es importante, pues en los 
fenómenos sociales las variables suelen estar autocorrelacionadas, como, por ejemplo, en la sustentabilidad para este caso en particular. Así, la confiabilidad por consistencia interna se hace con estimaciones de equivalencia de los componentes entre sí, y el coeficiente alpha de Cronbach es un estimador de equivalencia.

Tabla 5. Alpha de Cronbach para las respuestas de entrada y salida del cuestionario propuesto

\begin{tabular}{|c|c|c|c|c|c|}
\hline & & \multicolumn{2}{|c|}{ Por dimensiones } & \multicolumn{2}{|c|}{ Todas las variables } \\
\hline & & Entrada & Salida & Entrada & Salida \\
\hline \multirow{4}{*}{ Social } & s1 & 0,5680 & 0,6521 & 0,9437 & 0,9310 \\
\hline & $\mathrm{s} 2$ & 0,6918 & 0,7048 & 0,9409 & 0,9332 \\
\hline & s3 & 0,6485 & 0,7257 & 0,9435 & 0,9321 \\
\hline & s4 & 0,6734 & 0,8012 & 0,9423 & 0,9296 \\
\hline \multirow{8}{*}{ Ambiental } & a1 & 0,8984 & 0,8579 & 0,9402 & 0,9298 \\
\hline & a2 & 0,8866 & 0,8683 & 0,9407 & 0,9313 \\
\hline & a3 & 0,9009 & 0,8645 & 0,9431 & 0,9307 \\
\hline & a4 & 0,8900 & 0,8741 & 0,9399 & 0,9313 \\
\hline & a5 & 0,8898 & 0,8862 & 0,9379 & 0,9338 \\
\hline & a6 & 0,8820 & 0,8742 & 0,9370 & 0,9324 \\
\hline & a7 & 0,8910 & 0,8827 & 0,9367 & 0,9319 \\
\hline & a8 & 0,9008 & 0,8719 & 0,9414 & 0,9300 \\
\hline \multirow{8}{*}{ Económica } & e1 & 0,9309 & 0,8660 & 0,9380 & 0,9325 \\
\hline & e2 & 0,9227 & 0,8996 & 0,9381 & 0,9369 \\
\hline & e3 & 0,9300 & 0,8541 & 0,9413 & 0,9309 \\
\hline & e4 & 0,9237 & 0,8515 & 0,9395 & 0,9298 \\
\hline & e5 & 0,9187 & 0,8735 & 0,9367 & 0,9338 \\
\hline & e6 & 0,9150 & 0,8452 & 0,9386 & 0,9298 \\
\hline & e7 & 0,9202 & 0,8447 & 0,9377 & 0,9303 \\
\hline & e8 & 0,9192 & 0,8560 & 0,9389 & 0,9315 \\
\hline \multicolumn{2}{|c|}{ Total dimensión social } & 0,7109 & 0,7799 & & \\
\hline \multicolumn{2}{|c|}{ Total dimensión ambiental } & 0,9048 & 0,8868 & & \\
\hline \multicolumn{2}{|c|}{ Total dimensión económica } & 0,9317 & 0,8778 & & \\
\hline \multicolumn{2}{|c|}{ Todas las variables } & & & 0,9427 & 0,9349 \\
\hline
\end{tabular}

Fuente. Elaboración propia con base en las encuestas realizadas a los asistentes al taller y los cálculos de los autores.

En la tabla 5 se encuentra el estimador alpha de Cronbach para las respuestas de entrada — con 32 observaciones - y de salida — con 27- para cada pregunta del cuestionario. Se encontró en todas que el signo es positivo. Se estimó el coeficiente para las tres dimensiones de emprendimiento sustentable social, ambiental y económica-. En las últimas líneas se dan los resultados para cada dimensión y se deben comparar con el estimador de cada variable o ítem.
En las tres dimensiones el estimador obtenido fue superior a 0,7 , lo cual se considera aceptable. Si diera mayor o igual a 0,9 sería excelente y si da mayor a 0,8 es bastante bueno. De esta manera, la información obtenida permite confiar en el modelo, máxime cuando se sabe que es característico que al tenerse pocas variables el estimador tiende a ser más pequeño, como en el caso de la dimensión social que tiene apenas cuatro ítems. 
Al quitar alguna de las variables agrupadas, el estimador alpha de Cronbach del grupo disminuiría, situación que no es deseable. Por ejemplo, si en las mediciones de entrada se quitara la variable s1 de la dimensión social, el alpha de Cronbach de esa dimensión disminuiría a 0,56 y empeoraría la situación, pues al ser incluida tal variable con las otras tres el alpha es 0,71. Siguiendo este razonamiento, para el caso en cuestión es preciso afirmar que no conviene eliminar ninguna variable, ni a la entrada ni a la salida, pues en la inmensa mayoría de los casos el alpha caería, o, de acuerdo con los resultados, el beneficio en confiabilidad sería marginal y se perdería información.

\subsection{Seis meses después de la experiencia, ‘̇el cambio prevalece?}

Como complemento del estudio anterior se muestran los resultados del instrumento aplicado seis meses después del taller para confirmar si los cambios de percepción que se evidenciaron en el instrumento se convirtieron en aspectos culturales permanentes a tener cuenta a la hora de crear empresa. Se indaga si el emprendimiento sustentable se posicionó en la mente de los asistentes al taller.
Se construyen tres preguntas en las cuales cada una tiene tres opciones de emprendimiento sustentable - correspondientes a variables incluidas en el cuestionario de entrada y salida - y tres opciones vinculadas más estrechamente a una visión de emprendimiento sin mayores consideraciones de sustentabilidad. Se formularon las preguntas indicando que se escojan solo tres opciones. Se considera que, al escogerse por lo menos una opción de las incluidas en el formulario de entrada y salida, ya se entendería que hay mayor apropiación cultural de la sustentabilidad en el individuo. Es preciso notar que todas las opciones son válidas y hacen referencia al emprendimiento. En las tablas 6,7 y 8 se presentan los porcentajes de las opciones más escogidas por los asistentes al taller.

Las cuatro opciones más escogidas en la pregunta 1 señalan apropiación, pues tres de ellas corresponden a las respuestas relacionadas con el concepto de sustentabilidad (véase la Tabla 6).

Tabla 6. Respuestas pregunta 1 del cuestionario seis meses después

\begin{tabular}{|c|c|}
\hline Escoja tres de las seis opciones. Las ideas más importantes para la creación de mi nueva empresa son: & $\begin{array}{l}\text { Participación }(\%) \\
\text { Opción escogida* }\end{array}$ \\
\hline $\begin{array}{l}\text { Protección de ecosistemas en peligro: desde análisis de origen y procedencia hasta administración de residuos } \\
\text { —dimensión ambiental_ }\end{array}$ & $47,83 \%$ \\
\hline $\begin{array}{l}\text { Integración del emprendimiento en la planificación regional y local: alineación y protección con el territorio } \\
\text { —dimensión económica- }\end{array}$ & $47,83 \%$ \\
\hline $\begin{array}{l}\text { Debe existir pleno equilibrio entre la estabilidad económica del negocio, el menor impacto negativo al medio } \\
\text { ambiente y la responsabilidad social — concepto de sustentabilidad- }\end{array}$ & $56,52 \%$ \\
\hline $\begin{array}{l}\text { La idea de negocio debe tener criterios de innovación que hagan de ella un producto o servicio muy rentable } \\
\text { — concepto tradicional de emprendimiento- }\end{array}$ & $56,52 \%$ \\
\hline $\begin{array}{l}\text { Vender productos y servicios de excelente calidad, respetando siempre la normatividad tributaria, ambiental y } \\
\text { de seguridad y salud en el trabajo — concepto tradicional de emprendimiento- }\end{array}$ & $30,43 \%$ \\
\hline $\begin{array}{l}\text { La idea de negocio debe ser financieramente sólida y sostenible en el tiempo para garantizar mi estabilidad } \\
\text { económica y la de todos los que en ella trabajen — concepto tradicional de emprendimiento- }\end{array}$ & $43,48 \%$ \\
\hline
\end{tabular}

Fuente. Elaboración propia con base en encuestas realizadas a los asistentes al taller. 
Respecto a las motivaciones que se tienen a la hora de crear empresa, la más escogida — $82,61 \%$ — hace parte de la dimensión económica de la sustentabilidad. Los análisis del emprendimiento se centraron en las motivaciones como necesidad de logro, inherentes al emprendedor nato. Esta respuesta apunta a perfilar los asistentes al taller como personas con iniciativa hacia la creación de empresa con apropiación de sustentabilidad (véase la Tabla 7).

Tabla 7. Respuestas pregunta 2 del cuestionario seis meses después

\begin{tabular}{|l|c|}
\hline \multicolumn{2}{|c|}{ Escoja tres opciones. Mis principales motivaciones para iniciar un proyecto empresarial: } \\
\hline Ofrecer un producto o servicio que ayude a otros en su calidad de vida —dimensión económica— & $82,61 \%$ \\
\hline Controlar la intensidad de uso en los recursos naturales —dimensión económica— & $17,39 \%$ \\
\hline $\begin{array}{l}\text { No afectar el acceso de los residentes del lugar donde funcionará la empresa, a los principales recursos del } \\
\text { lugar y la región. Presente y futuro —dimensión social_ }\end{array}$ & $34,78 \%$ \\
\hline $\begin{array}{l}\text { Evitar que mi perfil sea de «empleado» y más bien aportar con el incremento de empleos —concepto } \\
\text { tradicional de emprendimiento— }\end{array}$ & $26,09 \%$ \\
\hline Estabilidad económica para mí y mi familia en el futuro — concepto tradicional de emprendimiento— & $60,87 \%$ \\
\hline Realización personal y profesional — concepto tradicional de emprendimiento— & $65,22 \%$ \\
\hline
\end{tabular}

Nota. Las expresiones entre guiones se muestran aquí con carácter explicativo, pero no fueron mostradas cuando se aplicó la encuesta.

Fuente. Elaboración propia con base en las encuestas realizadas a los asistentes al taller.

Al ser la variable de la dimensión social una de las tres más escogidas, se evidencia la apropiación del concepto de sustentabilidad más allá de lo económico-rentable. Es relevante la inclusión de los otros dos aspectos escogidos por los asistentes al taller, al ser medulares en la formación de empresa (véase la Tabla 8).

Tabla 8. Respuestas pregunta 3 del cuestionario seis meses después

\begin{tabular}{|l|c|}
\hline \multicolumn{2}{|c|}{ Escoja tres opciones. Lo que planificaría primero: } \\
\hline Efectos socioculturales en la comunidad de mi propuesta de emprendimiento —dimensión social— & $60,87 \%$ \\
\hline Oferta de trabajo bajo las premisas del empleo justo —dimensión económica_ & $21,74 \%$ \\
\hline $\begin{array}{l}\text { Gestión de residuos sólidos, tratamiento de aguas residuales y mínimo impacto en la contaminación atmosférica } \\
\text { —dimensión ambiental_ }\end{array}$ & $52,17 \%$ \\
\hline $\begin{array}{l}\text { Precios, ventas, análisis de mercado, marketing, presentación del producto, estrategias de publicidad —concepto } \\
\text { tradicional de emprendimiento— }\end{array}$ & $69,57 \%$ \\
\hline $\begin{array}{l}\text { Costos de producción, unidades a producir, márgenes de rentabilidad — concepto tradicional de } \\
\text { emprendimiento_- }\end{array}$ & $34,78 \%$ \\
\hline $\begin{array}{l}\text { Fuentes para la financiación de los recursos necesarios para la puesta en marcha —concepto tradicional de } \\
\text { emprendimiento- }\end{array}$ & $56,52 \%$ \\
\hline
\end{tabular}

Nota. Las expresiones entre guiones se muestran aquí con carácter explicativo, pero no fueron mostradas cuando se aplicó la encuesta.

Fuente. Elaboración propia con base en encuestas realizadas a los asistentes al taller.

En la población estudiada las decisiones de emprendimiento solían estar concentradas en la dimensión económica y ahora presentan indicios de actitudes hacia la sustentabilidad, sin embargo, para las generaciones futuras, las dimensiones de emprendimiento sustentable deberán ser irrenunciables al crear empresa. Los resultados sugieren que en los procesos de formación de los futuros profesionales se utilicen modelos como el desarrollado en esta investigación, los cuales incluyen instrumentos y experiencias que puedan coadyuvar, desde la didáctica y la pedagogía, a fortalecer los conceptos en emprendimiento sustentable. 


\section{Conclusiones}

$\mathrm{E}$ modelo de diagnóstico de la cultura emprendedora sustentable involucra sensibilización, apropiación y reflexión. A partir de la revisión teórica de los conceptos de cultura, emprendimiento y sustentabilidad se propuso el modelo presentado en esta investigación, en el que se reúnen las dimensiones ambiental, económica y social.

Los resultados del perfil de cultura emprendedora sustentable, provenientes del uso del instrumento a la entrada y salida de la actividad, permitieron estimar el perfil de cultura emprendedora sustentable y arrojaron lo siguiente: las variables ambientales en la entrada $-3,43-$ y la salida $-3,42$ - presentan mayor puntuación y prácticamente ningún cambio. En la dimensión social a la entrada se puntúa 3,42 y 3,29 a la salida, mostrándose aquí el mayor cambio entre las dimensiones. La dimensión económica inicia con la menor puntuación - 3,34-y es la única de las tres dimensiones que aumenta a la salida $-3,37-$.

Por tanto, el taller generó aumento en la puntuación de la dimensión económica, mientras que es notoria la mayor disminución en la dimensión social. Se encuentra así en esta una mayor dispersión en las respuestas de salida — más diferencias de opinión-, mientras que en las otras dos dimensiones las dispersiones en las respuestas de salida disminuyeron Las puntuaciones a la salida del taller implican mayor sentido crítico-reflexivo y, por tanto, mayor prudencia al responder las preguntas.

La validación del cuestionario mediante el alfa de Cronbach confirma la consistencia interna y la confiabilidad del instrumento, así como los resultados del modelo.

Seis meses después del taller se confirmó la existencia de actitudes hacia el emprendimiento sustentable y la apropiación del concepto, con lo cual se va más allá de lo económico-rentable y se avanza en el desarrollo y fortalecimiento de la cultura emprendedora sustentable.
El uso de estas herramientas y modelos invita a fortalecer los procesos de innovación en los currículos de las universidades al incorporar más actividades de aprender-haciendo hacia el desarrollo de cultura emprendedora sustentable que involucren su evaluación y apropiación en el mediano y largo plazo.

Como propuestas de continuidad se abren más oportunidades de aplicación del instrumento en otros contextos y poblaciones, de manera que aumente el número de participantes en las actividades. Se invita también a utilizar el instrumento en el inicio y en la salida de talleres con otros enfoques temáticos afines a la creatividad y la innovación.

\section{Referencias}

Acs, Z. J.; Amorós, J. E. (2008). Introduction: the startup process. Estudios de Economía, 35 (2), 121-132. DOI: https://doi. org/10.4067/S0718-52862008000200001

Amabile, T. (2005). Cómo matar la creatividad. En Harvard Business School Press (Comp.) Creatividad e innovación. (132). Barcelona: Deusto.

Arroyave-Puerta, A.; Marulanda-Valencia, F. (2019). Ecoemprendimiento, sostenibilidad y creación de valor. Revista Escuela de Administración de Negocios, (87), 1-26. DOI: https://doi.org/10.21158/01208160.n87.2019.2411

Audretsch, D. B.; Keilbach, M. C.; Lehmann, E. E. (2006). Entrepreneurship and economic growth. Oxford: Oxford University Press. DOI: https://doi.org/10.1093/ acprof:oso/9780195183511.001.0001

Barro, R. (1991). Economic growth in a cross section of countries. The Quarterly Journal of Economics, 106(2), 407-443. DOI: https://doi.org/10.2307/2937943

Blancas-Peral, F.; González-Lozano, M.; Guerrero-Casas, F.; Lozano-Oyola, M. (2010). Indicadores sintéticos de turismo sostenible: una aplicación para los destinos turísticos de Andalucía. Revista Electrónica de Comunicaciones y Trabajos de Asepuma, 11, 85-118. Recuperado de https://bit.ly/3vOPylx

Bugg-Levine, A.: Emerson, J. (2011). Jed impact investing: transforming how we make money while making a difference. Innovations Review, 6(3), 9-18. DOI: https://doi. org/10.1162/INOV_a_00077 
Callejón, M. (2009). La economía emprendedora de David Audretsch. Investigaciones regionales, (15), 47-54.

Castellanos, O. F.; Chávez, R. D.; Jiménez, C.N. (2003). Propuesta de formación en liderazgo y emprendimiento. Innovar, 1(22), 145-156.

Clegg, B. (2014). Negocios sustentables. México D. F.: Trillas.

Contreras-Pacheco, O.; Pedraza-Avella, A. C.; Martínez-Pérez, M. J. (2017). La inversión de impacto como medio de impulso al desarrollo sostenible: una aproximación multicaso a nivel de empresa en Colombia. Estudios Gerenciales, 33(142),13-23. DOI: https://doi.org/10.1016/j.estger.2017.02.002

Cortés-Mura, H. G. y Peña-Reyes, J. I. (2015). De la sostenibilidad a la sustentabilidad. Modelo de desarrollo sustentable para su implementación en políticas y proyectos. Revista Escuela Administración de Negocios, (78), 40-55. DOI: https://doi. org/10.21158/01208160.n78.2015.1189

Florida, R.; Mellander, C.; Stolarick, K. (2008). Inside the black box of regional development-human capital, the creative class and tolerance. Journal of Economic Geography, 8(5), 615-649. DOI: https://doi.org/10.1093/jeg/lbn023

Gómez-Roldán, I. (2013). Innovación y cultura organizacional (tesis de doctorado). Universidad Nacional de Educación a Distancia UNED, España. Recuperado de https://bit. ly/3nWN7aA

Gómez-Roldán, I.; García-Norato, O.; Melo-Perdomo, S., Orrego, C. (2019). Cultura emprendedora empresarial. Caso: estudiantes administración de empresas seccional Ubaté. En I. Gómez-Roldán; O. M. García-Norato (Coords.) Cultura emprendedora empresarial en la Universidad de Cundinamarca-Colombia. (37-64) Fusagasugá: Universidad de Cundinamarca. Recuperado de https://bit.ly/3ttNqe3

Hernández-Sampieri, R.; Fernández-Collado, C.; Baptista-Lucio, M. (2014). Metodología de la investigación. México D. F.: Mcgraw-Hill.

List, R.; Rodríguez, P., Pelz-Serrano, K.; Benítez-Malvido, J.; Lobato, J. M.; (2017). La conservación en México: exploración de logros, retos y perspectivas desde la ecología terrestre. Revista Mexicana de Biodiversidad, 88, 65-75. DOI: https://doi.org/10.1016/j.rmb.2017.10.007

Martínez-García, I.; Padilla-Carmona, M. T.; Suárez-Ortega, M. (2019). Aplicación de la metodología Delphi a la identificación de factores de éxito en el emprendimiento. Revista de Investigación Educativa, 37(1), 129-146. DOI: https://doi.org/10.6018/rie.37.1.320911

Muñoz, P. (2013). The distinctive importance of sustainable entrepreneurship. Current Opinion in Creativity, Innovation and Entrepreneurship, 2(1),1-6. DOI: https://doi.org/10.11565/ cuocient.v2i1.26

Organización de las Naciones Unidas. (1987). Informe Comisión Mundial Sobre Medio Ambiente y Desarrollo (A/42/427). Recuperado de https://bit.ly/3vHbA6u
Oriol, P.; van Klink, A.; Rozo-Grisales, J.A. (2011). Create impact! Handbook for sustainable entreperneurship. Enviu-Innovators in Sustainability. Recuperado de https://bit.ly/3heHLGj

Petit-Torres, E. E. (2007). La gerencia emprendedora innovadora como catalizador del emprendimiento económico. Revista de Ciencias Sociales, XIII(3), 495-506.

Pomering, A. A.; Kyriazis, E.; Johnson, L. W. (2014). Building sustainability into services marketing: expanding decision-making from a mix to a matrix. Ponencia presentada en Agents of change: Proceedings of the ANZMAC Annual Conference. Brisbane, QLD, Australia, 1-3 de diciembre.

Restrepo-Sánchez, L. (2018). Nuevos hallazgos de la supervivencia $y$ crecimiento de las empresas en Colombia. Bogotá: Confecámaras. Recuperado de https://bit.ly/3vO3E3b

Rodríguez-Moreno, D. C. (2016). Emprendimiento sostenible: significado y dimensiones Revista Katharsis, (21), 419-448. DOI: https://doi.org/10.25057/25005731.775

Rodríguez-Ramírez, A. (2009). Nuevas perspectivas para entender el emprendimiento empresarial. Revista Pensamiento y Gestión, (26), 94-119. Recuperado de https://bit. ly/3f5EXIQ

Ruiz-Ruano, A. M.; Puga, J. (2016). Emprendimiento sostenible en la universidad y valores ambientales. Psyecology, 7(1), 1-24. DOI: https://doi.org/10.1080/21711976.2015.1114218

Shepherd, D.; Patzelt, H. (2011). The new field of sustainable entrepreneurship: studying entrepreneurial action linking «what is to be sustained» with «what is to be developed». Entrepreneurship Theory and Practice Journal, 35(1), 137-163. DOI: https://doi.org/10.1111\%2Fj.15406520.2010.00426.x

Timmons, J.; Zacharakis, A.; Spinelli, S. (2004). Business plans that work. Nueva York: McGraw-Hill.

Vásquez-Stanescu, C. L.; Carrillo-Ozal, A. G.; Tona-Castillo, M. E.; Galíndez-Jiménez, M. V.; Macias-Camacho, K.A.; Esposito de Díaz, C. (2017). Sistema de gestión energética y ambiental de Productos Alimex CA. Suma de Negocios, 8(18), 115-121. DOI: https://doi.org/10.1016/j.sumneg.2017.11.003

Velásquez-Álvarez, L. V.; Vargas-Hernández, J. G. (2012). La sustentabilidad como modelo de desarrollo responsable y competitivo. Ingeniería de Recursos Naturales y del Ambiente, (11), 97-107.

Zisis, N.; Moya, P.; Molina, F. (2017). Percepciones de académicos sobre las dificultades para el fomento de la innovación y el emprendimiento: el caso de la FCFM de la Universidad de Chile. Journal of Technology Management \& Innovation, 4(12), 97-105. DOI: https://doi.org/10.4067/ S0718-27242017000400010 